\title{
Physician's guide to the diagnosis, treatment, and follow-up of inherited metabolic diseases
}

\author{
Editors: Nenad Blau, Marinus Duran, K. Michael Gibson and Carlo Dionisi-Vici
}

Christian Staufner • Verena Peters

(C) SSIEM 2014

This book combines and updates two previous editions, Physician's Guide to the Treatment and Follow-Up of Metabolic Diseases and Physician's Guide to the Laboratory Diagnosis of Metabolic Diseases. It is a unique source of information on the diagnosis, treatment, and follow-up of patients with inherited metabolic diseases. More than 500 conditions, grouped according to disorder category, are described. The structure of the book was redesigned, new disorders are added, and most chapters have a uniform layout. The first part of each chapter provides a compact introduction, biochemical pathways, signs and symptoms, reference and pathological values, diagnostic algorithms, flow charts, and recommendations for confirmatory tests. The second part describes detailed treatment options and recommendations for follow-up.

One of the strengths of this guide is the combination of diagnosis and therapy in one single volume. Further, much of the information is presented in tables which gives a good overview and makes the information easily readable. Indices, such as Disorder Index, Test and Medication Index, and Sign Symptoms Index facilitate the usage of this guide. In comparison to the previous editions, new chapters address emergency diagnostic procedures, newborn screening, genetic counseling, and the SSIEM Classification. As in the previous edition, the chapters about the most important biochemical tests provide very useful tables listing metabolites and their corresponding disorders together with explanations on interpretation and pitfalls.

The authors are acknowledged experts from across the world, and the book supplies clinicians and clinical biochemists with data that should facilitate the diagnosis and treatment of patients with inherited metabolic diseases.
C. Staufner $\cdot$ V. Peters $(\bowtie)$

Division of Inborn Metabolic Diseases, Department of Pediatrics, University Children's Hospital Heidelberg, Heidelberg, Germany

e-mail: Verena.Peters@med.uni-heidelberg.de

C. Staufner

e-mail: Christian.Staufner@med.uni-heidelberg.de 